\title{
Large-scale cell production of stem cells for clinical application using the automated cell processing machine
}

\author{
Daisuke Kami ${ }^{1}$, Keizo Watakabe ${ }^{2}$, Mayu Yamazaki-Inoue ${ }^{3}$, Kahori Minami ${ }^{3}$, Tomoya Kitani ${ }^{4}$, Yoko Itakura ${ }^{5}$,
} Masashi Toyoda ${ }^{5}$, Takashi Sakurai ${ }^{2}$, Akihiro Umezawa ${ }^{3}$ and Satoshi Gojo ${ }^{1 *}$

\begin{abstract}
Background: Cell-based regeneration therapies have great potential for application in new areas in clinical medicine, although some obstacles still remain to be overcome for a wide range of clinical applications. One major impediment is the difficulty in large-scale production of cells of interest with reproducibility. Current protocols of cell therapy require a time-consuming and laborious manual process. To solve this problem, we focused on the robotics of an automated and high-throughput cell culture system. Automated robotic cultivation of stem or progenitor cells in clinical trials has not been reported till date. The system AutoCulture ${ }^{\varpi}$ used in this study can automatically replace the culture medium, centrifuge cells, split cells, and take photographs for morphological assessment. We examined the feasibility of this system in a clinical setting.
\end{abstract}

Results: We observed similar characteristics by both the culture methods in terms of the growth rate, gene expression profile, cell surface profile by fluorescence-activated cell sorting, surface glycan profile, and genomic DNA stability. These results indicate that AutoCulture ${ }^{\varpi}$ is a feasible method for the cultivation of human cells for regenerative medicine.

Conclusions: An automated cell-processing machine will play important roles in cell therapy and have widespread use from application in multicenter trials to provision of off-the-shelf cell products.

Keywords: Automated cell culture system, Cell transplantation, Stem cells, Clinical trial, Cell processing facility

\section{Background}

Degenerative diseases affect increasing numbers of people, particularly in developed countries with aging populations. Despite advancements in medicine, modalities to cure advanced diseases are often not available. Therefore, regenerative therapy may become the standard treatment option in cardiovascular medicine. Recent developments in stem cell biology, including those related to induced pluripotent stem cells (iPSCs) and tissue-derived stem/ progenitor cells, are a giant leap toward the goal. Recently, myocardium-derived stem/progenitor cells were isolated by several institutes [1-3]. These cell populations have the potential to repair the diseased heart, and clinical trials are currently ongoing.

\footnotetext{
* Correspondence: gojos@koto.kpu-m.ac.jp

'Department of Regenerative Medicine, Kyoto Prefectural University of Medicine, 465 Kajii-cho, Kawaramachi-Hirokoji, Kamigyo-ku, Kyoto 602-8566, Japan

Full list of author information is available at the end of the article
}

In tandem with these developments in stem cell biology and the large number of completed and ongoing clinical trials, attempts have been made to commercialize these therapies [4]. The most prominent therapeutic strategy is cell transplantation. However, harvested cells or tissues are usually limited in quantity and stem cells properties may vary from batch to batch, hindering the reliability for clinical applications. Moreover, current cell therapy protocols are laboratory centered and labor intensive, requiring highly skilled personnel and weeks to months to harvest sufficient quantities of stem/progenitor cells from the isolated tissues. These manual procedures are expensive and can result in high phenotypic and yield variability between different trials and institutions [5].

Strategies to validate advanced medicinal products have been established; however, these "best practices" still depend on the ability of personnel to perform them, such as the cultivation of stem/progenitor cells under strictly

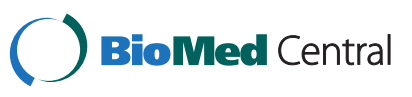


controlled conditions [6]. High process reproducibility can be achieved by automation, and several effective automatic cell culture systems have been reported [7-12]. These automated platforms have the potential to provide costeffective, large-scale expansion of stem/progenitor cells with consistent phenotype for clinical use and improved operational safety [13]. Progress in robot platforms for cell culture has resulted in several prototypes to implement large-scale expansion and harvesting of stem/progenitor cells with yield and phenotypic reproducibility. An automated culture system by "The Automation Partnership Biosystems (TAP Biosystems)" has cultivated human embryonic stem cells and bone marrow-derived cells [14,15]. Kawasaki Heavy Industries (Tokyo, Japan) has created AutoCulture $^{\odot}$ (Additional file 1), which can automate many manual steps in cell culture, including media exchange, centrifugation of cells, splitting and passaging, and recording of cell morphology (Figure 1A). To the best of our knowledge, no cell products obtained from an automated culture apparatus have actually been transplanted into humans for regenerative therapy.
Our institute recently completed a phase I clinical trial using autologous cardiac stem cells (CSCs) isolated by manual cell culture techniques to treat ischemic cardiomyopathy [16]. The trial is registered in the Japanese government database for clinical trials using human stem cells and ClinicalTrials.gov, which is a world-wide registry and results database for clinical trials involving humans, as AutoLogous Human CArdiac-Derived Stem Cell to Treat Ischemic cArdiomyopathy (ALCADIA; Identifier: NCT00981006). CSCs are manually cultivated by a single experienced investigator for approximately 1 month to minimize variability of the final cell products. To advance this trial from a single-center to a multi-center randomized trial, we evaluated AutoCulture ${ }^{\oplus}$ by comparing the growth rate, morphology, and phenotype of cells cultivated using this method with those of manually cultured CSCs.

\section{Results}

Cellular morphology and growth

Calculations based on the net cell number and doubling time obtained in the ALCADIA trial (Additional file 2)
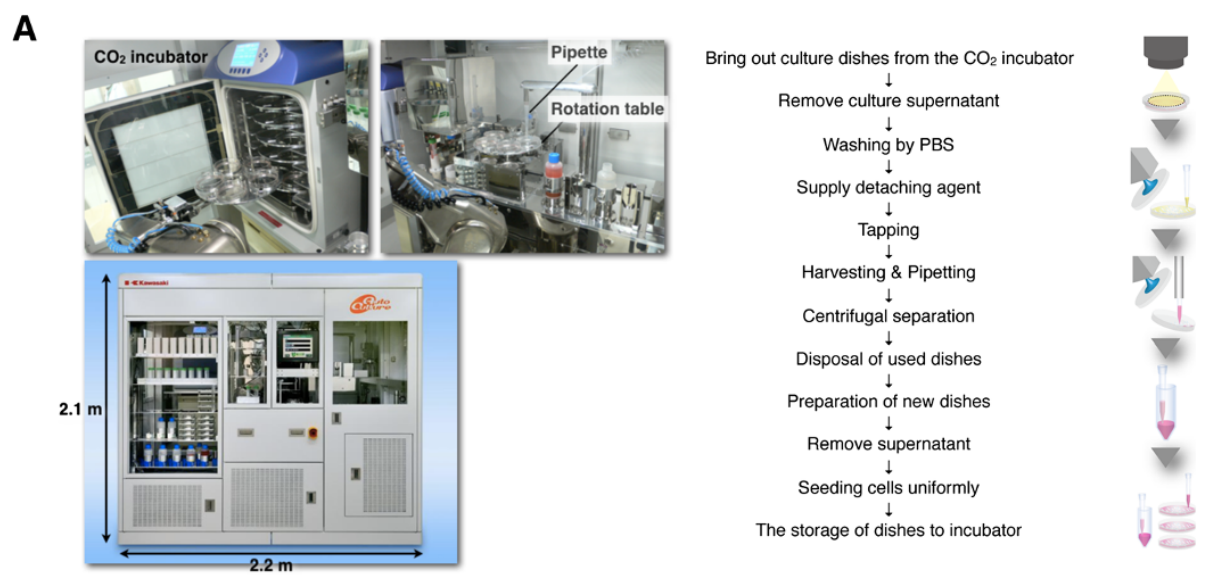

$\mathbf{B}$

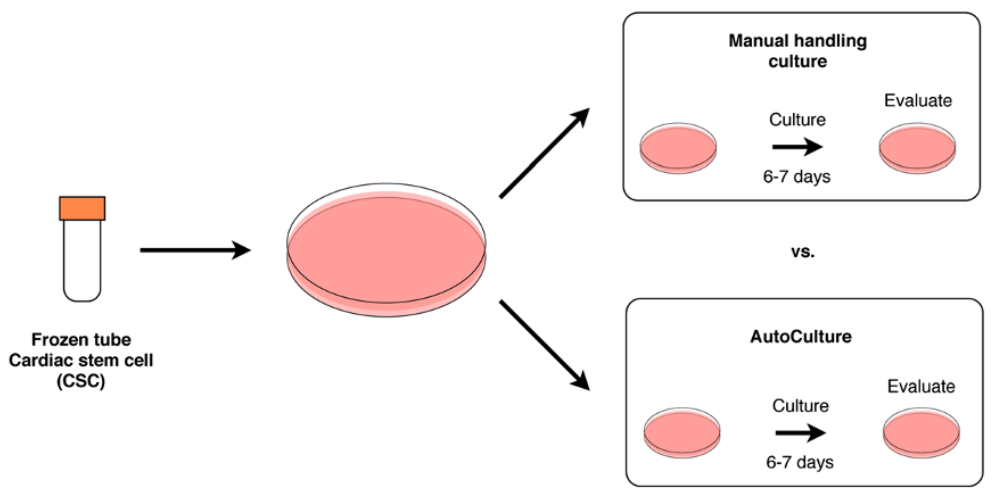

Figure 1 Process maps showing the key steps in human cardiac stem cell culture using manual methods and the AutoCulture ${ }^{\circledR}$ system. (A) The automated culture system AutoCulture ${ }^{\oplus}$ by Kawasaki Heavy Industries (left) can automate every step of manual cell culture under current good manufacturing practice (CGMP) grade. (B) Schematic representation of the experiment. Human cardiac stem cells (CSCS) were thawed and split into 2 dishes for either manual culture or automated culture using AutoCulture ${ }^{\oplus}$. 
indicated that a culture duration of 2 weeks was sufficient to obtain the appropriate cell number for clinical trial when cells after the second passage (P2) were used as the starting material. Identically seeded culture plates were maintained manually or by automation using AutoCulture ${ }^{\circledast}$ (Figure 1B). The morphology of CSCs cultured using the automated system was similar to that of manually cultured CSCs on day 7 and 14 after seeding (Figure 2A). Under both the conditions, the cells were of similar size, exhibited a low nucleus/cytoplasm ratio, and had a spindle-like shape. In addition, the growth rate was not significantly different, as indicated by cell counts at passage (Figure 2B). Trypan blue staining revealed no significant difference in cell viability between the culture methods. Moreover, both the methods effectively washed out the cells, as indicated by the paucity of adherent cells on discarded culture dishes (data not shown). These results suggest that manual passage was effectively replicated using AutoCulture ${ }^{\odot}$.

\section{Gene expression}

To investigate the gene expression profiles, RT-PCR analysis was performed according to the shipping criteria for cultivated cells in the current clinical trial (ALCADIA). We examined expression levels of the pluripotency related genes NANOG, OCT4, SOX2, and REX1 and 2 transcription factor genes involved in cardiomyocyte development, NKX2.5 and GATA4 (Figure 2C). The stem cell markers OCT4, REX1, and GATA4 were expressed by both cell populations; however, neither NANOG nor NKX2.5 expression was detectable. Moreover, expression levels were not significantly different between the 2 groups on either day 7 or day 14.

\section{Cell surface marker expression profiles}

Cell surface markers indicative of CSCs and other phenotypes were detected by fluorescence-activated cell sorting (FACS) (Figure 3A). Under both the culture conditions, the cells were positive for the mesenchymal stem cell (MSC) markers CD29 and CD90 and the vascular endothelial marker CD105 and negative for the hematopoietic lineage marker CD45 and MHC class II. In addition, fluorescent intensities measured by FACS were similar for all positive markers, indicating that equal proportions of cells in both the populations expressed these proteins. Moreover, almost all the cells were CD29 positive, whereas at least 2 populations were distinguished on the basis of CD90 expression. Furthermore, STRO-1, which is expressed by mesenchymal stem cells in the bone marrow, was negative in both the populations. Although the surface expression profiles of CSCs and bone marrow-derived stem cells overlap, STRO1 expression can discriminate cardiac MSCs from bone marrow-derived MSCs.

\section{Surface glycan expression profile by lectin microarray analysis}

Recently, glycan expression profiling has been reported to be an effective cell validation tool to complement phenotype analysis by epigenetic and gene expression analyses

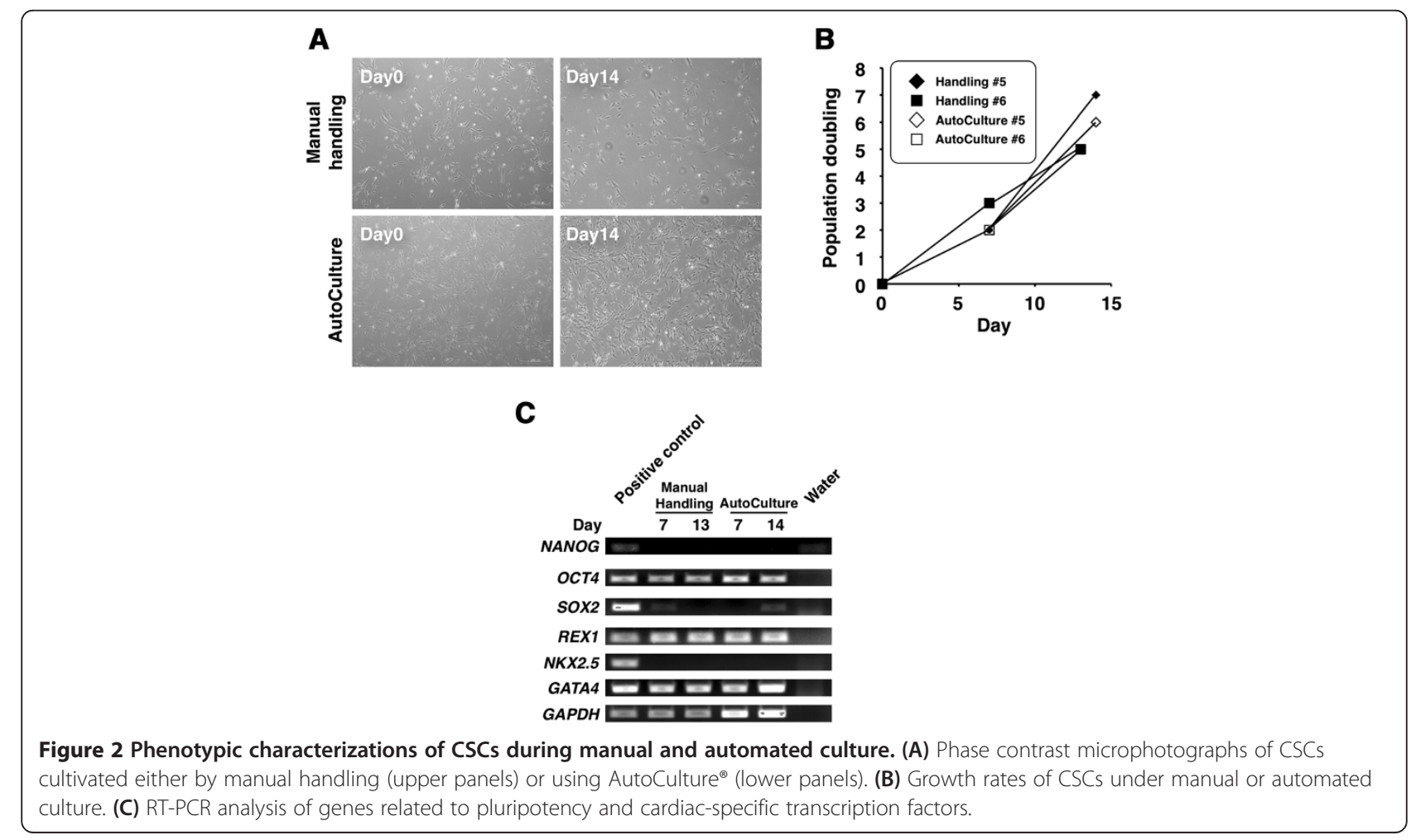




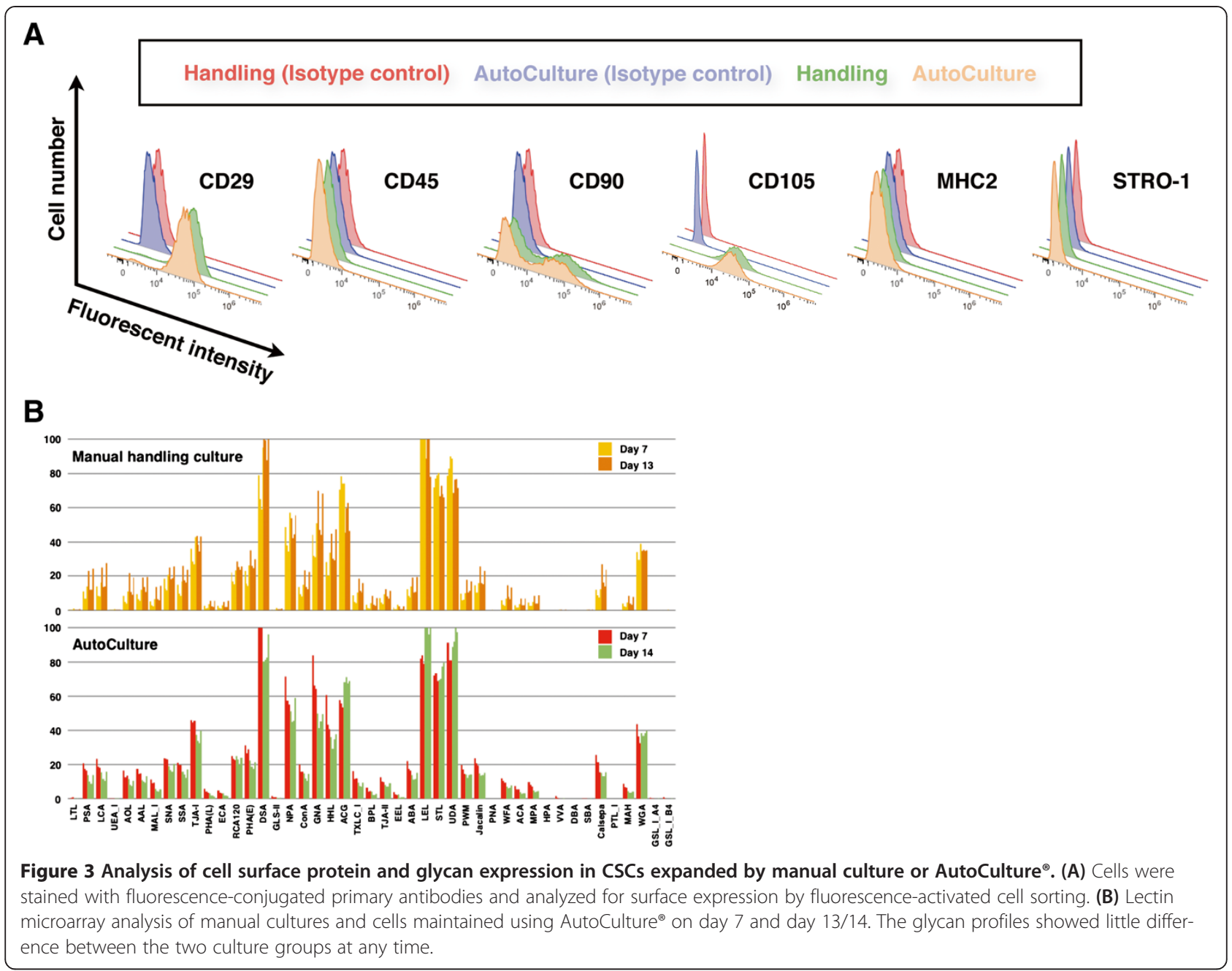

[17]. These lectin profiles showed similar patterns, and no significant differences in expression intensities were observed between the 2 culture groups on either day 7 or day 13/14 after seeding (Figure 3B). The washing process used to harvest adherent cells may have profound effects on the cell surface structure and expression. CSCs harvested from the AutoCulture ${ }^{\curvearrowleft}$ system exhibited similar surface expression profiles and overall viability to those cultured manually.

\section{Analysis of array comparative genomic hybridization (aCGH) and microarray}

To detect genomic DNA mutations on AutoCulture ${ }^{\oplus}$, we performed aCGH analysis (Agilent technologies) on day 7 and day 14 and compared them with the tissue derived from human right atrial appendage (RAA) (Figure 4A). There were no differences in genomic DNA mutation between CSCs in AutoCulture ${ }^{ø}$ and RAA. To investigate the global gene expression profile changes between CSCs in manual culture and CSCs in AutoCulture ${ }^{\bullet}$, we performed a pairwise comparison of gene expression microarray data using NIA array analysis [18]. The results revealed a similar gene pattern between them (Figure 4B, Additional file 3). The "Symbol" of 162 gene probes was left blank in 258 overexpressed gene probes.

\section{Discussion}

Cell-based regenerative medicine is still in the early stages of development $[19,20]$. The quality of cells for transplant depends on the ability of skilled personnel to isolate, expand, and harvest cultured cells. For consistency of cell yield and phenotype, it is imperative that methodological consistency is strictly maintained. Automation greatly enhances the consistency of culture conditions and may thus reduce the variability in cell quality that is one of the great impediments to the widespread application of cell-based therapy till date. In this study, we used the automated cell culture system AutoCulture ${ }^{\circledast}$ to expand human CSCs isolated from the RAA for use in the ALCADIA clinical trial designed to assess the safety of cell-based therapy for patients with ischemic cardiomyopathy. RAA-derived cells containing CSCs exhibited similar growth rates and gene 


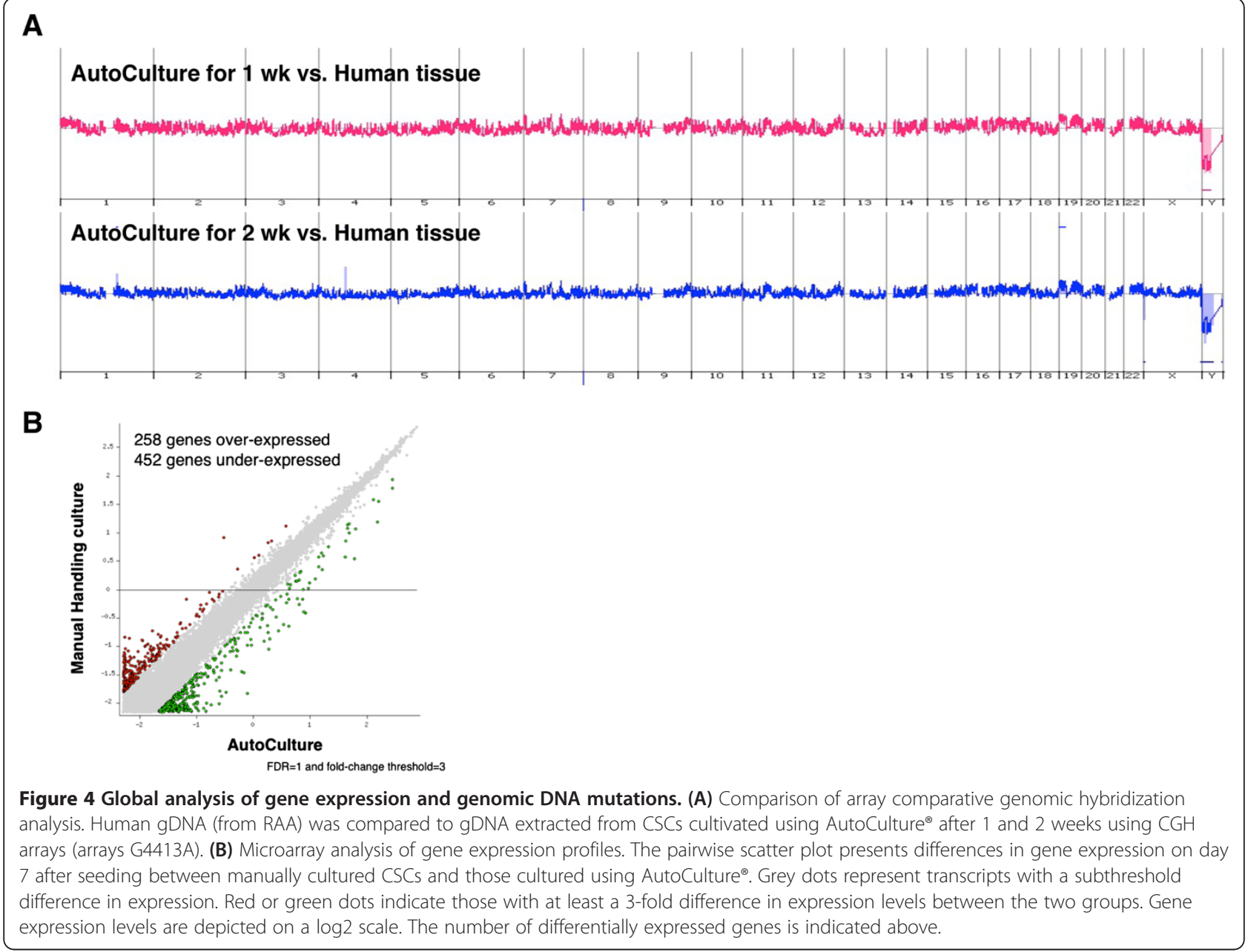

expression profiles between manual and automated cultures. Thus, the AutoCulture ${ }^{\varpi}$ system effectively replicated manual culture and demonstrated scalability and stability in addition to safety and cost-effectiveness. Indeed, we found no significant differences in phenotype between the two culture methods. Cells in both the populations had similar morphologies, mean growth rates, and expression levels of genes associated with pluripotency and the mesenchymal lineage. In addition, the surface glycan profile was virtually identical, while aCGH analysis revealed no difference in genomic DNA mutation frequency. Finally, the approximately 41,000-probe Agilent Whole Human Genome Microarray chip G4112F showed that only approximately $1 \%$ of transcripts measured were significantly under- or overexpressed. The successful transfer of manual to automated cell culture may be attributable to the high flexibility of the machine, which can faithfully copy every step and condition, including media changes, splitting, and passaging in a controlled environment.

AutoCulture ${ }^{\circledast}$ is an all-in-one automated cell culture system consisting of robot arms, tube and flask decappers, flask holders, flask tappers, media pumps, a pipette head, a centrifugal separator, a rotating plate, and a $\mathrm{CO}_{2}$ incubator. In addition to media change and passage, it permits routine observation. To automate these culture steps, it is necessary to program the humidity, temperature, volume and flow of liquid, and robot arm motion that transfers flasks from or into the $\mathrm{CO}_{2}$ incubator or flask holder. Another automated cell culture platform, TAP CompacT, was also shown to be an effective system for culture of adherent cells by the Healthcare Engineering group [14]. However, the lack of a centrifugal separator in that system may result in differences between the manual and automated processes, possibly explaining why automation resulted in a smaller population of STRO-1+ cells and overall lower cell yield after the first passage [21]. STRO-1 expression is not a necessary or specific marker for stem or progenitor cells, and somatic stem cells may be more resistant to nutritional and chemical stress [22]. Residual trypsin in the culture media may have adversely affected the survival of differentiated cells, but it is not clear whether stem or progenitor cells can survive or not. On the other hand, the AutoCulture $^{\oplus}$ system efficiently removes trypsin/EDTA by washing and centrifugation. There were no significant 
differences in the surface marker expression profile or the mean rate of proliferation between these cells and those maintained manually, strongly suggesting that both populations of RAA-derived CSCs contain equal properties.

The AutoCulture ${ }^{\oplus}$ system can save labor and costs by expanding the scale of production and maintaining uniformity of results. In addition, this system can simultaneously cultivate different cells without cross-contamination because it can be equipped with a connecting hatch to multiple $\mathrm{CO}_{2}$ incubators. Large-scale production and multisample cell culture capacity for cell transplantation may be a prerequisite for commercialization of cell products under current good manufacturing practice (cGMP) grade. Production methods for cell therapy should be designed to ensure that the end product is standardized and safe. cGMP is a quality assurance system that ensures that the cell product meets preset specifications with minimal lot-to-lot variability [23]. It requires traceability of raw materials used in cell culture and validated standard operating procedures (SOPs) throughout the process [24,25]. Current good tissue practice (cGTP) is intended to prevent human cells, tissues, and cellular and tissue-based products from contamination by infectious disease agents and to ensure that these cells and tissues maintain their integrity and function. The controlled environment of a carefully designed, constructed, validated, and maintained clean room will minimize the risks of environmental contamination and decrease the possibility of cross-contamination [26]. Based on cGMP, aseptic handling and filling of raw materials should be performed in a grade A environment (class 100) with a grade B background (class 1,000). Clean room disciplines, gowning procedures, cleaning programs, and maintenance of air handling units are included in SOPs. Environmental monitoring is essential in clean room quality control. Proper cleaning, maintenance, repair, and attire are major issues for cGMP [27].

Construction and maintenance of a cGMP facility is so expensive that it may be difficult to conform to these standards on a large scale without automation. Unlike manual culture, the robots enabled the environment in the cell culture cabinet to be completely separated from the external environment. Moreover, automated cell culture machines can be equipped with cleaning and monitoring systems to prevent contamination by microorganisms and cross-contamination by other cell types cultured in tandem. These properties may meet the stringent conditions for a human cell processing facility while reducing both construction and maintenance costs.

In Japan, the regulatory path of a regenerative cell therapy using this automated machine will be to obtain an approval for the end products, such as cells or tissues, based on the new guidelines and philosophy at an initial phase. An important requirement for obtaining approval is publication of the safety and reliability of the machine to produce the final biological products in a peer-reviewed journal. The similar properties of cell products between those obtained by machine and those obtained by manual culture, as demonstrated in this study, could support approval of a clinical trial using this machine, which is currently being planned.

\section{Conclusion}

AutoCulture $^{\circ}$ is one of the best candidates to solve the problems inherent in large-scale production and harvesting of human cells for clinical applications. The automated cell processing system can reproduce many complex operations performed by professional staff and can maintain multiple cell lines automatically. Thus, this automation system will be a powerful tool for both clinical trials exploring the potential of autologous or allogeneic cell-based regeneration therapies and for the commercialization.

\section{Methods}

\section{Isolation of human CSCs containing atrial appendage}

After this study was approved by the ethics committees of Tokyo Metropolitan Geriatric Hospital (ID: \#220106), human cardiac tissue samples from RAA were surgically excised from 7 patients (60-75 years old) during cardiac surgery. All patients provided written informed consent. A cell population containing CSCs was acquired according to the current protocol for ALCADIA [28]. In brief, the tissue fragments were cut into $5 \times 5-\mathrm{mm}$ pieces and incubated with $0.2 \%$ collagenase type II and $0.1 \%$ DNase I (Worthington Biochemicals) at $37^{\circ} \mathrm{C}$ for $30 \mathrm{~min}$. The cells were cultivated in a basic culture medium of Dulbecco's modified Eagle medium (DMEM)/F12 supplemented with $10 \%$ fetal bovine serum (FBS) and $40 \mathrm{ng} / \mathrm{ml}$ basic fibroblast growth factor (bFGF). The cells were seeded in 60mm dishes coated with collagen type I. The cultured cells were passaged twice, harvested, and frozen until used in this experiment. P2 cell population was utilized as the starting material for this comparison experiment.

\section{Cell expansion and harvesting}

After thawing, the cells derived from the human atrium were seeded at $1 \times 10^{5}$ cells per $100-\mathrm{mm}$ culture dish and cultivated for 5-7 days. The cells were split at 1:10 at $80 \%-90 \%$ confluence. The basic culture medium was replaced every 3 or 4 days. For automated culture, we used the same lot of CSCs. After seeding, the culture dishes were placed in the AutoCulture ${ }^{\odot}$ chamber and transferred into the internal $\mathrm{CO}_{2}$ incubator by the robot arm (Figure 1A, Additional file 4). For media replacement, the robot arm retrieved the culture dishes from the incubator and set them on a rotating table. The dish covers were removed by the robot arm, a specified amount of medium was discarded, fresh medium was supplied by a new pipette, the covers were returned, 
and the culture dishes was transferred back to the $\mathrm{CO}_{2}$ incubator. For passage, the old medium was removed and DPBS was pipetted onto the dishes under gentle shaking. After washing in DPBS, AutoCulture ${ }^{\circ}$ supplied trypsin, oscillated the culture dishes, and returned them to the $\mathrm{CO}_{2}$ incubator for a 5-min incubation. Following this, the robot arm moved the culture dishes onto the rotation table, added a prespecified volume of the basic culture media, and transferred the cell suspension from each dish to a separate $50-\mathrm{ml}$ centrifuge tube. The cell suspension was centrifuged at $200 \times g$ for $5 \mathrm{~min}$ at room temperature, and the supernatant was discarded. Fresh basic culture medium was supplied to the cell pellet, which was then resuspended. The washed cell suspension was subcultured at approximately 1:10 onto new culture dishes and returned to the $\mathrm{CO}_{2}$ incubator.

Reverse transcription-polymerase chain reaction (RT-PCR) Total RNA was extracted from cell populations containing CSCs, from human iPSCs, and raw human heart tissue samples using the RNeasy Plus Mini Kit (QIAGEN) as positive/negative control. Total RNA from human iPSCs and the human heart (Clontech Laboratories) was used as the positive control for each primer. Total RNA (500 ng per reaction) was converted to cDNA using the Transcriptor High Fidelity cDNA Synthesis Kit (Roche Applied Science) according to the manufacturer's protocol. Primers for the cardiacspecific transcription factors NKX2.5 and GATA4; the stem cell markers NANOG, OCT3/4, SOX2, and REX1; and the housekeeping gene GAPDH were obtained from PrimerBank (Additional file 5).

\section{Flow cytometric analysis}

The cells $\left(1 \times 10^{6}\right.$ per reaction) were stained in autoMACS Running Buffer (Miltenyi Biotec.) with fluorescenceconjugated primary antibodies for $30 \mathrm{~min}$ at $4^{\circ} \mathrm{C}$. The cells were then analyzed on the Attune Acoustic Focusing Cytometer (Applied Biosystem), and the data were analyzed using FlowJo 8.8.7 software (TOMY Digital Biology). Antibodies used for phenotyping included anti-human CD29PE, CD90-PE, CD105-FITC, STRO-1-FITC, CD45-PE, and $\mathrm{MHC}$ class II-PE. Isotype controls were FITCconjugated mouse $\operatorname{IgG}_{1}$, PE-conjugated mouse $\operatorname{IgG}_{1}$, and FITC-conjugated mouse IgM.

\section{Lectin microarray analysis}

Proteins were extracted from each cell population in hydrophobic and hydrophilic fractions using the CelLytic MEM Protein Extraction Kit (Sigma-Aldrich), as described previously [29]. Lectin microarray analysis was performed as described previously, with only minor modifications [30]. The glycoprotein (200 ng) was labeled with Cy3 monoreactive dye (GE Healthcare) in DPBS containing 0.5\%
Triton X-100 (PBSTx) at room temperature for $1 \mathrm{~h}$. The Cy3-labeled glycoprotein solution $(60 \mu \mathrm{l})$ was applied to the LecChip (GP Bioscience), which has triplicate spots specific for 45 lectins on each glass slide. An evanescent-field fluorescence scanner (GlycoStationTM Reader) was used to analyze the LecChip. All data were analyzed with GlycoStationTM Tools Signal Capture 1.0 and GlycoStationTM Tools Pro 1.0 software (GP Bioscience). To expand the dynamic range, the data were subjected to a gain-merging procedure, and the merged data were then normalized with max-normalization, as described previously [29].

\section{aCGH analysis}

Genomic DNA from the heart tissue and cultured cells was isolated using the DNeasy Blood \& Tissue Kit (QIAGEN). Labeled test and reference DNAs were combined, denatured, preannealed with Cot-1 DNA (Invitrogen) and blocking agent, and then hybridized to the arrays (SurePrint G3 Human CGH Microarray 2x400K, Agilent Technologies). After hybridization and washing, the arrays were scanned at 3- $\mu \mathrm{m}$ resolution using an Agilent G2505C scanner. Images were analyzed with Feature Extraction software 10.7.3.1 (Agilent Technologies) using the CGH 107 Sep09 protocol for background subtraction and normalization.

\section{Gene expression analysis}

Gene expression analysis was performed using the Agilent Whole Human Genome Microarray chip G4112F (Agilent Technologies), which contains $>41,000$ probes. Raw data were normalized and analyzed by GeneSpring GX11 software (Silicon Genetics). Pairwise scatter plot analysis of the global gene expression profiles of both manually cultured cells and autocultured cells was performed on day 7 after thawing. The number of differentially expressed genes is indicated over each scatter plot. The NIA Array [18] web tool was used for pairwise scatter plot analysis. Gene expression microarray data have been submitted under accession number GSE 44032. Analysis of microarray experiments was conducted using the Aberration Detection Method-2 statistical algorithm (Agilent Technologies) on the basis of the combined $\log 2$ ratios at a threshold of 6.0. The data were centralized, and calls with average $\log 2$ ratios $<0.3219$ were filtered to exclude false positives.

\section{Additional files}

Additional file 1: Document 1. Specialization of the automated cell processing machine (Auto Culture ${ }^{\circledR}$ ).

Additional file 2: Document 2. Quantitative cellular aspects for ALCADIA clinical trial.

Additional file 3: Table S1. Results of microarray analysis of CSCs in manual culture and AutoCulture ${ }^{\oplus}$. To investigate the differences in global gene expression profile between CSCs in manual culture and CSCs in AutoCulture $^{\circledast}$, we performed a pairwise comparison of gene expression 
microarray data using NIA array analysis. The results revealed similar gene expression patterns between them.

Additional file 4: Movie 1. AutoCulture ${ }^{\oplus}$. Movie of the culture robot in AutoCulture ${ }^{\oplus}$.

Additional file 5: Table S2. RT-PCR primer sequences. RT-PCR primer sequences were obtained from PrimerBank (http://pga.mgh.harvard.edu/ primerbank/).

\section{Abbreviation}

CSC: Cardiac stem cells; FACS: Fluorescence-activated cell sorting; MSCs: Mesenchymal stem cells; aCGH: Array comparative genomic hybridization; RAA: Right atrial appendage; cGMP: Current good manufacturing practice; SOPs: Standard operating procedures; cGTP: Current good tissue practice; bFGF: Basic fibroblast growth factor.

\section{Competing interests}

DK, MYI, KM, TK, YI, MT, AU and SG declare that they have no competing interests. KW and TS are employees of Kawasaki Heavy Industries, Ltd.

\section{Authors' contributions}

DK, MT, AU, and SG designed the research; DK, KW, YI, KM, MYI, and performed the experiments; DK, MT, and SG analyzed the data; and DK, TK, $\mathrm{YI}$, and SG wrote the manuscript. All authors read and approved the final manuscript.

\section{Acknowledgments}

We would like to express our sincere thanks to Nakata M. (Kyoto Prefectural University of Medicine) and Akutsu H (National Center for Child Health and Development). This research was supported by grants from Ministry of Education, Culture Sports, Science and Technology (MEXT) of Japan.

\section{Author details}

'Department of Regenerative Medicine, Kyoto Prefectural University of Medicine, 465 Kajii-cho, Kawaramachi-Hirokoji, Kamigyo-ku, Kyoto 602-8566, Japan. ${ }^{2}$ System Technology Development Center, Kawasaki Heavy Industries, Ltd., 3-1-1 Higashi Kawasaki-cho, Chuo-ku, Kobe 650-8670, Japan.

${ }^{3}$ Department of Reproductive Biology and Pathology, National Center for Child Health and Development, 2-10-1 Okura, Setagaya-ku, Tokyo 157-8535, Japan. ${ }^{4}$ Department of Cardiovascular Medicine, Kyoto Prefectural University of Medicine, 465 Kajii-cho, Kawaramachi-Hirokoji, Kamigyo-ku, Kyoto 602-8566, Japan. ${ }^{5}$ Department of Vascular Medicine, Tokyo Metropolitan Institute of Gerontology, 35-2 Sakae-cho, Itabashi-ku, Tokyo 173-0015, Japan.

Received: 7 May 2013 Accepted: 12 November 2013

Published: 15 November 2013

\section{References}

1. Takehara N, Tsutsumi Y, Tateishi K, Ogata T, Tanaka H, Ueyama T, Takahashi T, Takamatsu T, Fukushima M, Komeda M, et al: Controlled delivery of basic fibroblast growth factor promotes human cardiosphere-derived cell engraftment to enhance cardiac repair for chronic myocardial infarction. J Am Coll Cardiol 2008, 52(23):1858-1865.

2. Smith RR, Barile L, Cho HC, Leppo MK, Hare JM, Messina E, Giacomello A, Abraham MR, Marban E: Regenerative potential of cardiosphere-derived cells expanded from percutaneous endomyocardial biopsy specimens. Circulation 2007, 115(7):896-908.

3. Rota M, Padin-Iruegas ME, Misao Y, De Angelis A, Maestroni S, FerreiraMartins J, Fiumana E, Rastaldo R, Arcarese ML, Mitchell TS, et al: Local activation or implantation of cardiac progenitor cells rescues scarred infarcted myocardium improving cardiac function. Circ Res 2008, 103(1):107-116.

4. Vogel G: Stem cells for sale. Science 2010, 330(6008):1173.

5. Tran CA, Burton L, Russom D, Wagner JR, Jensen MC, Forman SJ, DiGiusto DL: Manufacturing of large numbers of patient-specific $T$ cells for adoptive immunotherapy: an approach to improving product safety, composition, and production capacity. J Immunother 2007, 30(6):644-654.

6. Soncin S, Lo Cicero V, Astori G, Soldati G, Gola M, Surder D, Moccetti T: A practical approach for the validation of sterility, endotoxin and potency testing of bone marrow mononucleated cells used in cardiac regeneration in compliance with good manufacturing practice. J Trans/ Med 2009, 7:78.

7. Joannides A, Fiore-Heriche C, Westmore K, Caldwell M, Compston A, Allen $\mathrm{N}$, Chandran S: Automated mechanical passaging: a novel and efficient method for human embryonic stem cell expansion. Stem Cells 2006, 24(2):230-235

8. Kino-Oka M, Ogawa N, Umegaki R, Taya M: Bioreactor design for successive culture of anchorage-dependent cells operated in an automated manner. Tissue Eng 2005, 11(3-4):535-545.

9. Terstegge S, Laufenberg I, Pochert J, Schenk S, Itskovitz-Eldor J, Endl E, Brustle O: Automated maintenance of embryonic stem cell cultures. Biotechnol Bioeng 2007, 96(1):195-201.

10. Thomas RJ, Anderson D, Chandra A, Smith NM, Young LE, Williams D, Denning C: Automated, scalable culture of human embryonic stem cells in feeder-free conditions. Biotechnol Bioeng 2009, 102(6):1636-1644.

11. Koike H, Kubota K, Sekine K, Takebe T, Ouchi R, Zheng YW, Ueno Y, Tanigawa N, Taniguchi H: Establishment of automated culture system for murine induced pluripotent stem cells. BMC Biotechnol 2012, 12:81.

12. Thomas RJ, Chandra A, Liu Y, Hourd PC, Conway PP, Williams DJ: Manufacture of a human mesenchymal stem cell population using an automated cell culture platform. Cytotechnology 2007, 55(1):31-39.

13. Hubbell JA, Palsson BO, Papoutsakis ET: Preface: tissue engineering and cell therapies: II. Biotechnol Bioeng 1994, 43(8):683.

14. Thomas R, Chandra A, Hourd P, Williams D: Cell culture automation and quality engineering: a necessary partnership to develop optimized manufacturing processes for cell-based therapies. J Assoc Lab Autom 2008, 13(3):152-158.

15. Thomas RJ, Hourd PC, Williams DJ: Application of process quality engineering techniques to improve the understanding of the in vitro processing of stem cells for therapeutic use. J Biotechnol 2008, 136(3-4):148-155.

16. Takehara N, Ogata T, Nakata M, Kami D, Nakamura T, Matoba S, Gojo S, Sawada T, Yaku H, Matsubara H: The ALCADIA (Autologous Human CardiacDerived Stem Cell to Treat Ischemic Cardiomyopathy) trial, Circulation. PHILADELPHIA, PA: LIPPINCOTT WILLIAMS \& WILKINS 530 WALNUT ST; 2012:19106-3621. USA: 2783-2783.

17. Toyoda M, Yamazaki-Inoue M, Itakura Y, Kuno A, Ogawa T, Yamada M, Akutsu H, Takahashi Y, Kanzaki S, Narimatsu H, et al: Lectin microarray analysis of pluripotent and multipotent stem cells. Genes Cells 2011, 16(1):1-11.

18. Sharov AA, Dudekula DB, Ko MS: A web-based tool for principal component and significance analysis of microarray data. Bioinformatics 2005, 21(10):2548-2549.

19. Ptaszek LM, Mansour M, Ruskin JN, Chien KR: Towards regenerative therapy for cardiac disease. Lancet 2012, 379(9819):933-942.

20. Gojo S, Toyoda M, Umezawa A: Tissue engineering and cell-based therapy toward integrated strategy with artificial organs. J Artif Organs 2011, 14(3):171-177

21. Liu $Y$, Hourd $P$, Chandra A, Williams DJ: Human cell culture process capability: a comparison of manual and automated production. J Tissue Eng Regen Med 2010, 4(1):45-54.

22. Kuroda Y, Kitada M, Wakao S, Nishikawa K, Tanimura Y, Makinoshima H, Goda M, Akashi H, Inutsuka A, Niwa A, et al: Unique multipotent cells in adult human mesenchymal cell populations. Proc Natl Acad Sci USA 2010, 107(19):8639-8643.

23. Unger C, Skottman H, Blomberg P, Dilber MS, Hovatta O: Good manufacturing practice and clinical-grade human embryonic stem cell lines. Human Mol Genet 2008, 17(R1):R48-R53.

24. Allport-Settle MJ: Good Manufacturing Practice (GMP) Guidelines. Raleigh: Pharmalogika; 2009.

25. Burger SR: Current regulatory issues in cell and tissue therapy. Cytotherapy 2003, 5(4):289-298.

26. USP: 32-NF 27 Cell and gene therapy products. United States Pharmacopeia, Rockville: Manufacturing of cell therapy products; 2008.

27. Niazi SK: Sterile products, vol. 6. London: Informa Healthcare Inc; 2009

28. Takehara NOT, Nakata M, Kami D, Matoba NT, Gojo S, Sawada T, Yaku H, Matsubara H: The ALCADIA (autologous Human Cardiac-derived Stem Cell To Treat Ischemic Cardiomyopathy) Trial, Late Breaking Clinical Trial Application. Los Angeles: American Heart Association, Scientific Session; 2012.

29. Kuno A, Itakura Y, Toyoda M, Takahashi Y, Yamada M, Umezawa A, Hirabayashi J: Development of a data-mining system for differential 
profiling of cell glycoproteins based on lectin microarray. J Proteom Bioinfo 2008, 1(2):5.

30. Itakura Y, Kimura M, Gojo S, Toyoda M, Kami D, Motomura N, Umezawa A, Kyo $S$, Ono M: Glycan profiling using a lectin microarray is a novel validation tool for monitoring the damage to freeze-thawed cells. Low Temp Med 2011, 37:7.

doi:10.1186/1472-6750-13-102

Cite this article as: Kami et al.: Large-scale cell production of stem cells for clinical application using the automated cell processing machine. BMC Biotechnology 2013 13:102.

\section{Submit your next manuscript to BioMed Central} and take full advantage of:

- Convenient online submission

- Thorough peer review

- No space constraints or color figure charges

- Immediate publication on acceptance

- Inclusion in PubMed, CAS, Scopus and Google Scholar

- Research which is freely available for redistribution 